\title{
Fatores associados às barreiras para a prática de atividade física de adolescentes
}

\author{
Factors associated with barriers for practice of physical activity in \\ adolescents
}

Geraldo Jose Ferrari Junior ${ }^{1}$, Rubian Diego Andrade', Cleber Fernando Rebelatto', Thais Silva Beltrame ${ }^{1}$, Andreia Pelegrini ${ }^{1}$, Érico Pereira Gomes Felden

\section{Resumo}

O objetivo deste estudo foi analisar as barreiras que discriminam os adolescentes em ativos e insuficientemente ativo. Ainda descrever os fatores associados com tais barreiras em meninos e meninas com baixos níveis de atividade física. Participaram 312 adolescentes (10-16 anos) de Florianópolis-SC. Foram coletadas informações sociodemográficas, de percepção de saúde, estresse, status social na escola e na educação física, sonolência diurna excessiva, atividade física, além da percepção de barreiras para a prática da atividade física. As meninas apresentam maior frequência de barreiras percebidas $(57,2 \%)$. As barreiras que discriminavam as meninas insuficientemente ativas "não tenho como me deslocar para onde posso praticar", "preguiça” e "em casa ninguém faz". Essas barreiras foram associadas à sonolência diurna excessiva $(\mathrm{p}=0,001)$, baixa percepção de status social na escola ( $\mathrm{p}=0,003)$ e baixa percepção de estresse $(p=0,046)$. Entre os meninos, observou-se diminuição do número de barreiras percebidas conforme aumento da idade $(\mathrm{r}=-0,175 ; \mathrm{p}=0,039)$. As barreiras que discriminavam meninos insuficientemente ativos: "amigos moram longe", "preguiça” e "não me sinto motivado". Tais barreiras foram associadas aos fatores: ser mais jovem, baixa percepção de estresse e de status social na Educação Física. As barreiras para a prática de atividade física diferem-se entre meninos e meninas insuficientemente ativos. No entanto, a preguiça esteve associada em ambos os sexos. Além disso, diferentes fatores dificultam o envolvimento de adolescentes para a prática de atividade física. Contudo, os baixos níveis de estresse estiveram associados às barreiras para a prática de atividade física de meninos e meninas.

\section{Palavras-chave}

Atividade motora; Adolescente; Saúde do adolescente.

\begin{abstract}
The aim of this study was to analyze the barriers that discriminate adolescents in active and insufficiently active. Also describe the factors associated with such barriers in boys and girls with low levels of physical activity. Participated 312 adolescents (1016 years) from Florianópolis, SC. We collected sociodemographic information, health perception, stress, social status at school and physical education, excessive daytime sleepiness, physical activity, beyond the perception of barriers to physical activity. girls have the bighest frequency of perceived barriers (57.2\%). The barriers that discriminate against insufficiently active girls "do not have to move me to where I can practice", "lazy" and "at home no one does." These barriers were associated with excessive daytime sleepiness ( $p=0.001)$, low perceived social status at school $(p=0.003)$ and low perception of stress $(p=0.046)$. Among boys, there was a decrease in the number of barriers perceived as increasing age ( $r=-0.175$; $p=0.039$ ). The barriers that discriminate insufficiently active boys were: friends live far away", "lazy" and "did not feel motivated." Such barriers were associated with factors: being younger, low perception of stress and social status Physical Education. Barriers to physical activity differ between boys and girls insufficiently active. However, laziness was associated in both sexes. Moreover, different factors binder the involvement of adolescents to practice physical activity. However, low levels of stress were associated with barriers to physical activity in boys and girls.
\end{abstract}

\section{Keywords}

Motor Activity; Adolescent; Adolescent Health.

\section{Introdução}

A prática de atividade física é importante na prevenção de diversas complicações à saúde. Além disso, estudos mostram a importância da atividade física no desenvolvimento das funções cognitivas superiores em crianças e adolescentes ${ }^{1,2}$. Neste sentido, o estímulo ao maior envolvimento com as práticas motoras deve ser iniciado nas fases anteriores à vida adulta, como evidenciou o estudo de Azevedo et al. ${ }^{3}$ 
ao verificar que adultos mais ativos tiveram maior envolvimento com as práticas esportivas na adolescência.

Contudo, pesquisas ${ }^{4-6}$ indicam que poucos jovens atingem as recomendações para atividade física. No Brasil, no último levantamento de âmbito nacional realizado pelo $\mathrm{IBGE}^{7}$ a prevalência de adolescentes ativos fisicamente foi de 30,1\%. Durante a adolescência ocorrem diversas alterações maturacionais, comportamentais e socioculturais que podem influenciar na redução da participação em atividades físicas ${ }^{8}$. Em estudo de coorte realizado em Pelotas-RS com adolescentes identificou-se diminuição dos níveis de atividade física ao longo da adolescência e início da vida adulta ${ }^{9}$. Para Cumming et al. $^{10}$, em seu modelo biocultural, a maturação sexual possui efeitos diretos e indiretos para atividade física em adolescentes. Ainda, em estudo conduzido com adolescentes alemães, a idade cronológica apresentou influência na prática de atividade física ${ }^{11}$. Assim, parece que o envolvimento em prática regular de atividade física precisa ser melhor sistematizado com o avançar da idade, incluindo as práticas no âmbito escolar.

Ainda que estudo ${ }^{6}$ apresente barreiras associadas aos níveis insuficientes de atividade física, existe a necessidade de explorar os fatores associados a essas restrições e observar o comportamento dessas relações entre meninos e meninas, até mesmo conforme a idade. Diante dessas evidências, faz-se necessário um melhor entendimento de fatores pessoais e ambientais para possam se constituir em barreiras para um estilo de vida mais ativo ${ }^{9-10}$. Neste sentido, o objetivo do presente estudo foi identificar os fatores associados às barreiras para a prática da atividade física de adolescentes insuficientemente ativos. Além disso, foi meta desta pesquisa investigar o comportamento dessas relações entre meninos e meninas.

\section{Métodos}

A amostra deste estudo foi formada por 312 estudantes (53,2\% do sexo feminino) de 10 a 16 anos, matriculados regularmente no ensino fundamental. Utilizou-se uma amostragem do tipo intencional, por conveniência, em uma escola da rede pública municipal de Florianópolis - SC com aproximadamente, 330 alunos matriculados.

Inicialmente o projeto foi submetido ao Comitê de Ética em Pesquisa com Seres Humanos da UDESC (parecer $n^{\circ} 114015 / 2014$ ). Depois de ser aprovado, os pesquisadores entraram em contato com a Secretaria de Educação de Florianópolis, com o intuito de obter a autorização para realização da pesquisa. Após esta fase, os pesquisadores foram até uma escola, com o objetivo de explicar todos os detalhes da pesquisa para que a direção autorizasse a realização da mesma. Para minimizar possíveis vícios no processo de coleta de dados, recorreu-se a uma unidade escolar mais afastada do centro em que os alunos não estivessem habituados com pesquisas. Após o consentimento da gestão, estabeleceu-se uma parceria entre a unidade escolar e o Núcleo de Ciências da Saúde (NUPECIS) da UDESC. Dessa forma, a Universidade comprometeu-se em apresentar estratégias práticas para a melhoria dos níveis de atividade física e saúde dos escolares.

Todos os alunos que estavam frequentando as aulas regularmente foram convidados a participarem da pesquisa. A amostra final foi formada pelos alunos que assinaram o Termo de Assentimento e entregaram o Termo de Consentimento Livre e Esclarecido assinado pelos responsáveis. Esta coleta foi realizada em 2014, por uma equipe de pesquisadores, ocorrendo em sala de aula.

As informações para a pesquisa foram coletadas por meio de questionários. Para a análise da variável dependente do estudo utilizou-se um questionário elaborado por Santos et al. ${ }^{12}$. Este instrumento foi proposto para adolescentes de $14 \mathrm{a}$ 
17 anos. No entanto, com o objetivo de minimizar possíveis erros na interpretação das questões pelos adolescentes de 10 a 13 anos, foi realizado um estudo piloto para verificar a clareza e entendimento dos participantes. A avaliação das barreiras para atividade física em adolescentes contém doze questões que indicam motivos para a não prática de atividade física. As opções de resposta são apresentadas em uma escala Likert de quatro pontos, variando de "discordo muito" até "concordo muito". Para a análise, os adolescentes foram classificados quanto à presença da barreira ("concordo" ou "concordo muito") ou ausência ("discordo" e "discordo muito"). Ainda referente às análises, a quantidade de barreiras relatada por cada adolescente foi apresentada por mediana, diferença interquartil e a frequência relativa de cada uma das doze barreiras percebidas.

Foram coletadas informações de sexo, idade, turno escolar e nível socioeconômico. Devido ao número reduzido de participantes nas idades mais avançadas e para fins de análise, a idade foi agrupada em faixas etárias (10-11 anos, 12 e de 13-16 anos). Assim essa variável apresentou uma distribuição homogênea quanto ao número de participantes em cada grupo. O nível socioeconômico foi avaliado por meio do Critério de Classificação Econômica Brasil proposto pela Associação Brasileira de Empresas de Pesquisa ${ }^{13}$, o qual estima o poder aquisitivo das pessoas e famílias urbanas. A partir da soma dos pontos de cada questão, os indivíduos foram classificados em estratos econômicos (A, B1, B2, C1, C2, D e E). Da mesma forma que a idade, para fins de análise, esses estratos foram categorizados e considerou-se nível alto (A, B1), médio (B2 e C1) e baixo (C2, D e E).

O nível de atividade física foi investigado por meio do questionário proposto por Florindo et al. ${ }^{14}$. Este instrumento é composto por 17 questões sobre atividades físicas habituais, sendo considerado o tempo total em minutos e a frequência semanal. A validação concorrente deste questionário foi avaliada com o teste de corrida vai-e-vem de 20 minutos, pelo consumo máximo de oxigênio, frequência cardíaca e velocidade máxima. O processo de validação apresentou índices de concordância com o tempo total do teste $(\mathrm{r}=0,19)$, com a velocidade total para os meninos $(\mathrm{r}=0,20)$, e consumo máximo de oxigênio e tempo total para as meninas $(\mathrm{r}=0,17)$. Nas análises de reprodutibilidade, a correlação do escore semanal foi de 0,61 . Para as análises, os adolescentes foram classificados em insuficientemente ativos se realizassem menos de 300 minutos de atividades físicas semanais ${ }^{15}$.

A percepção de saúde foi avaliada por meio da questão "Como você avalia o seu estado de saúde atual?". Sendo realizada da mesma forma em estudo de Pereira et al. ${ }^{16}$. As opções de respostas foram: "excelente", "boa", "regular" e "ruim". Para fins de análise, estas respostas foram agrupadas em percepção positiva de saúde (excelente e bom) e negativa (regular e ruim).

$\mathrm{Na}$ avaliação do estresse, os adolescentes responderam à questão "Como você descreve o nível de estresse em sua vida?"16. As opções de respostas foram: "raramente estressado", "às vezes estressado", "quase sempre estressado" e "excessivamente estressado". Para fins estatísticos, as questões foram reagrupadas como alta percepção (excessivamente estressado e quase sempre estressado) e baixa percepção de estresse (às vezes estressado e raramente estressado).

Para avaliação da sonolência diurna excessiva, foi aplicada a Pediatric Daytime Sleepiness Scale (PDSS) ${ }^{17,18}$. A PDSS é composta por oito questões de múltipla escolha sendo que cada item é pontuado utilizando-se uma escala com variação de cinco categorias de resposta: 0- nunca, 1- quase nunca, 2- às vezes, 3- frequentemente, 4- sempre. Ao final quanto maior o somatório (máximo 32), maior a sonolência, quanto menor a pontuação (mínimo 0), menor a sonolência. Foi utilizada 
de forma dicotômica, sendo o $1^{\circ}$ e $2^{\circ}$ tercil considerado sem sonolência (até 15 pontos) e o $3^{\circ}$ tercil (16 pontos ou mais) como possível indicador de sonolência diurna excessiva.

Por fim, medidas antropométricas de massa corporal e estatura foram mensuradas seguindo procedimentos padronizados ${ }^{19}$. Estas medidas foram utilizadas para o cálculo do índice de massa corporal, o qual foi categorizado, por meio de pontos de corte estabelecidos pela literatura, classificando os adolescentes em eutróficos e excesso de peso.

A auto percepção de status social em relação à escola e a aula de Educação Física foi verificada por meio da Escala MacArthur de status social: versão para jovens proposta por Goodman et al. ${ }^{20}$. Esta é composta pela imagem de uma escada (escolha de 0 a 10 degraus) e no alto dela estão representados os alunos com as maiores notas, mais populares e respeitados, ou seja, com posição social mais elevada no ambiente escolar. Para fins de análise utilizou-se os tercis: $1^{\circ}$ tercil $(<7), 2^{\circ}$ tercil $(\geq 7$ - <8) e $3^{\circ}$ tercil $(\geq 8)$ para a percepção de status social na escola. Referente à adaptação da escala para o status social na Educação Física, no alto estariam os adolescentes mais habilidosos, com melhor desempenho nos esportes e primeiros escolhidos durante a seleção de times. Para análise da percepção de status social na educação física foi adotado como tercis: $1^{\circ}$ tercil $(<7), 2^{\circ}$ tercil $(\geq 7-<9)$ e $3^{\circ}$ tercil $(\geq 9)$.

A normalidade da distribuição dos dados foi testada em todas as variáveis por meio do teste de Kolmogorov-Smirnov, desta forma verificou-se em todas as variáveis uma distribuição não normal. Assim, a descrição da amostra, geral e de acordo com o sexo, foi realizada por meio da frequência, mediana e diferença interquartil. Devido às diferentes barreiras e fatores associados em meninos e meninas, optouse pela estratificação por sexo nas análises. Nas variáveis categóricas, recorreu-se ao teste Qui-quadrado para verificar as diferenças de proporções das características gerais, bem como, para a diferença das frequências de barreiras percebidas para a prática de atividade física entre sexo e na percepção de barreiras que discriminam fisicamente ativos ou não. Para identificar os fatores associados às barreiras em cada sexo recorreu-se ao teste de heterogeneidade do Qui-quadrado. Já para verificar diferenças entre os sexos nas variáveis contínuas idade e número de barreiras, foi utilizado o teste U de Mann-Whitney. Por fim, a correlação entre a idade e o número de barreiras percebidas, foi realizada pelo teste de Spearman. Para a análise dos dados foi utilizado o software The Statistical Package for the Social Sciences (SPSS) for Windows versão 20.0.

\section{Resultados}

Os dados indicaram diferenças significativas entre os sexos no nível de atividade física $(p<0,001)$, status social na escola $(p=0,004)$ e na aula de Educação Física $(\mathrm{p}=0,022)$ e no número de barreiras $(\mathrm{p}=0,003)$. Enquanto os meninos eram mais ativos fisicamente e se percebiam com menor status social na escola, as meninas apresentaram baixa percepção de status social na aula de Educação Física e possuíam maior média de barreiras percebidas para a prática de atividade física (Tabela 1).

As barreiras mais frequentes foram "falta de companhia para fazer atividade física" e "falta de lugares próximo de casa". As meninas apresentaram maior frequência de barreiras percebidas que os meninos em relação à "falta de lugares próximo de casa" $(\mathrm{p}=0,009)$, "clima” ( $\mathrm{p}=0,019)$, "falta de amigos" $(\mathrm{p}=0,022)$, "preguiça” ( $\mathrm{p}=0,043)$ e "falta de tempo" $(\mathrm{p}=0,012)$ (Tabela 2$)$. 
TABELA 1 - Características gerais dos adolescentes da amostra geral e estratificado por sexo.

\begin{tabular}{|c|c|c|c|c|}
\hline \multirow{2}{*}{ Variáveis } & Geral & Meninos & Meninas & \multirow{2}{*}{ p-valor } \\
\hline & $n=312$ & $n=146$ & $n=166$ & \\
\hline Idade, anos & $12,0(2,0)$ & $12,0(2,0)$ & $12,0(2,0)$ & $0,314^{*}$ \\
\hline \multicolumn{4}{|l|}{ Faixas etárias, \% } & \multirow{4}{*}{$0,573+$} \\
\hline $10-11$ & 30,9 & 28,8 & 32,7 & \\
\hline 12 & 27,0 & 26,0 & 27,9 & \\
\hline $13-16$ & 42,1 & 45,2 & 39,4 & \\
\hline \multicolumn{4}{|l|}{ Status de peso, $\%$} & \multirow{3}{*}{$0,185+$} \\
\hline Eutróficos & 90,2 & 87,8 & 92,4 & \\
\hline Excesso de peso & 9,8 & 12,2 & 7,6 & \\
\hline \multicolumn{4}{|l|}{ Nível socioeconômico, \% } & \multirow{4}{*}{$0,238+$} \\
\hline Alto & 17,4 & 16,6 & 18,2 & \\
\hline Médio & 58,7 & 63,4 & 54,5 & \\
\hline Baixo & 23,9 & 20,0 & 27,3 & \\
\hline \multicolumn{4}{|l|}{ Turno de estudo, \% } & \multirow{3}{*}{$0,079+$} \\
\hline Matutino & 70,5 & 75,3 & 66,3 & \\
\hline Vespertino & 29,5 & 24,7 & 33,7 & \\
\hline \multicolumn{4}{|l|}{ Atividade física, $\%$} & \multirow{3}{*}{$<0,001 \dagger$} \\
\hline Insuficientemente ativo & 51,0 & 39,0 & 61,4 & \\
\hline Ativo & 49,0 & 61,0 & 38,6 & \\
\hline \multicolumn{4}{|c|}{ Sonolência diurna excessiva, \% } & \multirow{3}{*}{$0,088+$} \\
\hline Possui & 39,8 & 34,7 & 44,2 & \\
\hline Não possui & 60,2 & 65,3 & 55,8 & \\
\hline \multicolumn{4}{|l|}{ Percepção de estresse, \% } & \multirow{3}{*}{$0,224 \dagger$} \\
\hline Alta & 15,2 & 12,5 & 17,5 & \\
\hline Baixa & 84,8 & 87,5 & 82,5 & \\
\hline \multicolumn{4}{|l|}{ Status social na escola, \% } & \multirow{4}{*}{$0,004 \uparrow$} \\
\hline Alto & 27,7 & 23,9 & 30,9 & \\
\hline Médio & 26,7 & 20,4 & 32,1 & \\
\hline Baixo & 45,6 & 55,6 & 37,0 & \\
\hline \multicolumn{4}{|c|}{ Status social na aula de educação física, \% } & \multirow{4}{*}{$0,022 \dagger$} \\
\hline Alto & 22,5 & 27,0 & 18,8 & \\
\hline Médio & 36,6 & 40,4 & 33,3 & \\
\hline Baixo & 40,8 & 32,6 & 47,9 & \\
\hline Número de barreiras, $\bar{x}$ & $4,0(4,0)$ & $3,0(4,0)$ & $4,0(4,0)$ & $0,003^{*}$ \\
\hline
\end{tabular}

Valores descritos em frequência ou mediana (diferença interquartil). $\dagger=p$-valor do teste de heterogeneidade do Qui-quadrado para variáveis categóricas. ${ }^{*}=\mathrm{p}$-valor do teste $U$ de Mann-Whitney para variáveis contínuas.

TABELA 2 - Frequência (\%) de barreiras percebidas para a prática de atividade física.

\begin{tabular}{llccc}
\hline Barreiras para prática da atividade física, \% & Geral & Meninos & Meninas & p-valor \\
\hline É difícil fazer atividade física sem alguma companhia. & 51,1 & 47,9 & 53,9 & 0,173 \\
\hline Não encontro lugares perto de casa com a atividade física que gosto. & 51,0 & 43,4 & 57,6 & 0,009 \\
\hline O clima dificulta minha prática de atividade física. & 37,8 & 31,2 & 43,4 & 0,019 \\
\hline É difícil fazer atividade física porque não conheço lugares perto de casa onde eu possa ir. & 37,3 & 32,2 & 41,8 & 0,052 \\
\hline Eu deixo de fazer atividade física porque prefiro fazer outra coisa. & 34,7 & 32,4 & 36,7 & 0,249 \\
\hline É difícil fazer atividade física porque não tenho como ir (ou voltar) onde posso praticar. & 30,8 & 31,0 & 30,7 & 0,529 \\
\hline É difícil fazer atividade física porque os amigos que me acompanham moram longe. & 30,2 & 24,1 & 35,4 & 0,022 \\
\hline Tenho preguiça de fazer atividade física & 27,6 & 22,5 & 31,9 & 0,043 \\
\hline É difícil fazer atividade física, porque em casa ninguém faz. & 26,6 & 26,8 & 26,5 & 0,531 \\
\hline Acho difícil fazer atividade física porque não me sinto motivado. & 24,8 & 24,6 & 24,8 & 0,537 \\
\hline Tenho muitas tarefas para fazer, por isso é difícil fazer atividade física. & 21,6 & 18,4 & 24,2 & 0,138 \\
\hline Falta tempo para fazer atividade física. & 19,2 & 13,4 & 24,1 & 0,012 \\
\hline
\end{tabular}

p-valor do teste de heterogeneidade do Qui-quadrado. 
A análise de frequência de barreiras identificou que 9,1\% da amostra considerou não possuir barreiras que dificultem a sua prática de atividade física (quantidade de barreiras percebidas $=0$ ).

$\mathrm{Na}$ análise de diferenças do número de barreiras, foram observadas diferenças entre os sexos apenas na faixa etária de 13-16 anos, as quais apontam que as meninas apresentam número de barreiras percebidas para a prática de atividade física superior em relação aos meninos $(\mathrm{p}=0,007)$ (Figura 1). Além disso, os resultados do teste de Spearman, não apresentado em tabela ou figura, entre a idade e o número de barreiras percebidas revelaram uma correlação de baixa magnitude, porém significativa e inversa no sexo masculino $(r=-0,175 ; p=0,039)$.

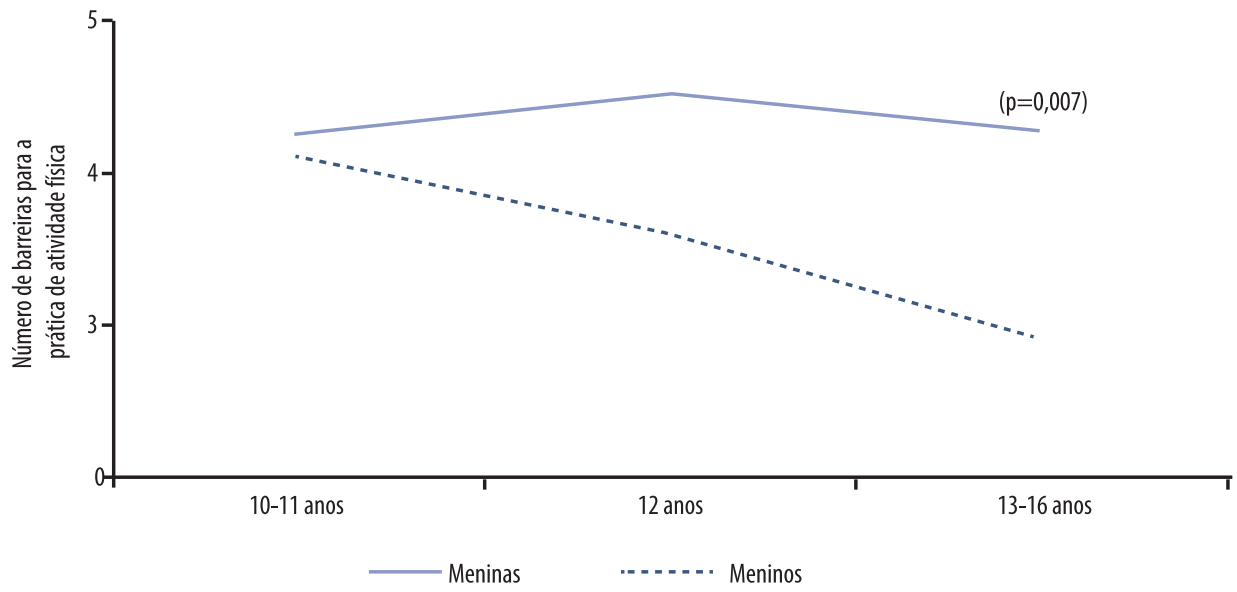

FIGURA 1 - Número de barreiras percebidas para a prática de atividade física de meninos e meninas de acordo com a faixa etária.

Quando analisada as frequências de percepção de barreiras que discriminam fisicamente ativos ou não, os meninos insuficientemente ativos apresentaram maior frequência das barreiras "amigos moram longe", "preguiça" e "não me sinto motivado". Para as meninas, as maiores frequências foram as barreiras "não tenho como me deslocar para onde posso praticar", "preguiça” e "em casa ninguém faz" (Tabela 3).

TABELA 3 - Frequência de percepção das barreiras para a prática de atividade física de meninos e meninas de acordo com o nível de atividade física.

\begin{tabular}{|c|c|c|c|c|c|c|}
\hline \multirow{3}{*}{ Barreiras para a prática de atividade física } & \multicolumn{3}{|c|}{ Meninos } & \multicolumn{3}{|c|}{ Meninas } \\
\hline & \multicolumn{2}{|c|}{ Fisicamente ativos } & \multirow{2}{*}{ p-valor } & \multicolumn{2}{|c|}{ Fisicamente ativas } & \multirow{2}{*}{ p-valor } \\
\hline & Sim & Não & & Sim & Não & \\
\hline É difícil fazer atividade física sem alguma companhia & 43,7 & 54,5 & 0,207 & 45,3 & 59,4 & 0,077 \\
\hline Não encontro lugares perto de casa com a atividade física que gosto & 40,9 & 47,3 & 0,455 & 64,1 & 53,5 & 0,180 \\
\hline O clima dificulta minha prática de atividade física & 33,3 & 27,8 & 0,489 & 37,5 & 47,1 & 0,226 \\
\hline É difícil fazer atividade física porque não conheço lugares perto de casa onde eu possa ir & 28,4 & 38,2 & 0,224 & 34,9 & 46,1 & 0,158 \\
\hline Eu deixo de fazer atividade física porque prefiro fazer outra coisa & 27,6 & 40,0 & 0,124 & 28,1 & 41,2 & 0,068 \\
\hline É difícil fazer atividade física porque não tenho como ir (ou voltar) de onde posso praticar & 27,6 & 36,4 & 0,271 & 15,6 & 40,2 & 0,001 \\
\hline É difícil fazer atividade física porque os amigos que me acompanham moram longe & 18,4 & 33,3 & 0,044 & 28,1 & 40,0 & 0,121 \\
\hline Tenho preguiça de fazer atividade física & 13,8 & 36,4 & 0,002 & 15,6 & 42,2 & $<0,001$ \\
\hline É difícil fazer atividade física, porque em casa ninguém faz & 23,0 & 32,7 & 0,202 & 17,2 & 32,4 & 0,031 \\
\hline Acho difícil fazer atividade física porque não me sinto motivado & 13,8 & 41,8 & $<0,001$ & 21,8 & 26,7 & 0,482 \\
\hline Tenho muitas tarefas para fazer, por isso é difícil fazer atividade física & 18,4 & 18,5 & 0,985 & 23,8 & 24,5 & 0,919 \\
\hline Falta tempo para fazer atividade física & 10,3 & 18,2 & 0,181 & 28,1 & 21,6 & 0,336 \\
\hline
\end{tabular}

p-valor do teste de heterogeneidade do Qui-quadrado. 
Os fatores associados às barreiras dos meninos foram: baixa percepção de estresse, ser mais jovens e baixa percepção de status social na Educação Física. Já os fatores associados às barreiras das meninas foram: sonolência diurna excessiva, baixa percepção de status social na escola e baixa percepção de estresse (Figura 2).

$\mathrm{n}(\%) \quad$ Barreiras $\quad$ Fatores Associados
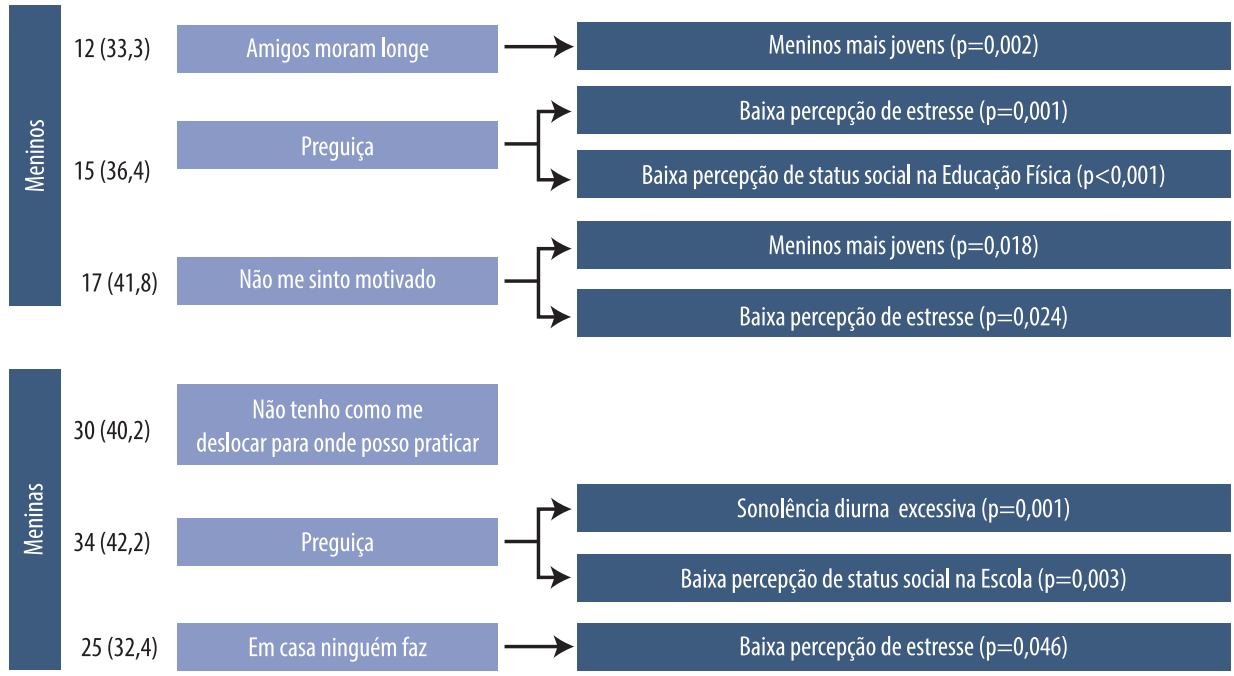

FIGURA 2 - Fatores associados às barreiras para a prática da atividade física dos adolescentes insuficientemente ativos, estratificado por sexo.

p-valor do teste de heterogeneidade do Qui-quadrado.

\section{Discussão}

Este estudo teve como objetivo identificar os fatores associados às barreiras para a prática da atividade física de adolescentes insuficientemente ativos. Além disso, investigar o comportamento dessas relações entre meninos e meninas. Em acordo com outras pesquisas ${ }^{6,21}$, os resultados do presente estudo mostraram que a percepção de barreiras para a prática de atividade física difere-se entre meninos e meninas insuficientemente ativos. No entanto, a preguiça foi a única barreira que esteve associada nos adolescentes em ambos os sexos. Além disso, os fatores que dificultam o envolvimento dos adolescentes na prática de atividade física também são diferenciados. Contudo, o "baixo nível de estresse" foi o único que esteve associado às barreiras para prática de atividade física de adolescentes insuficientemente ativos tanto de meninos quanto de meninas.

A baixa percepção de estresse esteve associada às barreiras "preguiça" e "não me sinto motivado" nos meninos e "em casa ninguém faz" nas meninas. Esta relação não foi investigada em estudos anteriores. Contudo, acredita-se que níveis muito baixos de estresse podem levar a baixa iniciativa do adolescente à prática de atividade física. Com isso, os meninos e meninas parecem necessitar de um nível de estresse moderado que seja provocado autonomia suficiente para que transcendam as barreiras associadas.

A única barreira percebida para a prática de atividade física que discriminou os insuficientemente ativos em ambos os sexos foi "ter preguiça". Corroborando com nosso estudo, outras pesquisas vêm relatando associação da preguiça aos baixos níveis de atividade física ${ }^{6,21}$, e destacam a necessidade de maior apoio social para incentivar a prática de atividade física entre adolescentes. Além disso, deve-se alertar que esta barreira vai além da força de vontade e preferências individuais ${ }^{6}$. A "preguiça", assim como comportamentos introspectivos, é comum nesta fase da vida, na qual o adolescente passa por alterações maturacionais e comportamen- 
tais. Um exemplo deste fenômeno entre os adolescentes é o atraso da inibição da secreção da melatonina durante o dia e o acúmulo mais lento durante a noite que provoca o atraso do início do sono dos adolescentes ${ }^{22}$. Essas mudanças biológicas têm repercussões significativas na sonolência diurna excessiva especialmente em adolescentes que estudam no período matutino ${ }^{22}$. Portanto, a barreira preguiça pode ser um reflexo dessa sonolência comum nesta fase da vida ${ }^{22}$.

Além disso, a sonolência diurna excessiva esteve associada à preguiça nas meninas. Recente revisão da literatura estabeleceu a relação entre a sonolência com a prática habitual de atividade física ${ }^{23}$. A sonolência diurna excessiva, caracterizada pelo aumento da necessidade de sono, "aparente preguiça” e desmotivação gerada pela estafa física e mental tem a sua origem em fatores endógenos e ambientais ${ }^{23}$. Quando entendida e devidamente tratada pode deixar de ser considerada uma barreira para comportamentos relacionados à saúde, incluindo a prática de atividade física.

Já nos meninos a barreira "preguiça" esteve associada à baixa percepção de status social na Educação Física. Os adolescentes com baixo status social na Educação Física se percebem como menos habilidosos e com pior desempenho nos esportes. Como muitas aulas de Educação Física são realizadas com a abordagem tecnicistas visando o rendimento esportivo, acredita-se que esta barreira "mascare" a vergonha do aluno menos habilidoso, tornando-se uma desculpa entre os colegas para não praticar atividade física. Desta forma, isso pode ocasionar a exclusão de alunos menos habilidosos, piores nos esportes e pouco aptos fisicamente ${ }^{24}$.

Apesar da limitação do agrupamento em faixas etárias e a maior concentração de idade (13,14, 15 e 16 anos) no grupo mais velho, verificou-se que o número de barreiras percebidas pelas meninas foi semelhante nas diferentes faixas etárias. Contudo, os rapazes mais velhos (13-16 anos) apresentaram perceber menos barreiras para a prática de atividade física que as meninas, o que é confirmado pela literatura $^{25,26}$. Resultados estes, possivelmente explicados considerando-se as diferenças socioculturais entre os sexos, como o maior envolvimento dos meninos em esportes e atividades vigorosas e das meninas em atividades de baixa intensidade ${ }^{25,27}$.

As barreiras que discriminavam as meninas insuficientemente ativas foram: "não tenho como me deslocar para onde posso praticar", "preguiça" e "em casa ninguém faz". Essas barreiras foram associadas à sonolência diurna excessiva, baixa percepção de status social na escola e baixa percepção de estresse. Sabe-se que especialmente no sexo feminino, pela maior vulnerabilidade às situações de risco, são extremante relevantes questões como acessibilidade, segurança pública, distância entre casa e locais de prática, companhia de amigos e/ou adultos para a prática ou não de atividade física. Assim discute-se que a presença desta barreira constitui-se não apenas como uma percepção a ser vencida intrinsicamente, mas como uma imposição social que muitas vezes coloca as meninas numa situação de inatividade não por escolha pessoal e sim por imposição.

Já entre os meninos, as barreiras que discriminavam os insuficientemente ativos foram: "amigos moram longe", "preguiça" e "não me sinto motivado". Tais barreiras foram associadas aos fatores: ser mais jovem, baixa percepção de estresse e baixa percepção de status social na Educação Física. A distância entre os colegas pode limitar a prática de atividade física juntos, supostamente, pelo fato dos meninos mais jovens necessitarem do auxílio dos pais para que se desloquem a um lugar comum, e seus pais não se disponibilizam para esse auxílio. Outros autores ${ }^{6}$ indicam que a motivação está ligada ao prazer, e se ofertado novas experiências, aumentando as opções de atividade física, a motivação dos adolescentes mais jovens aumenta com a possibilidade de vivências prazerosas durante as novas práticas. 
Das doze barreiras para atividade física a "falta de companhia" obteve maior frequência na amostra geral, resultado também apresentado por outros estu$\operatorname{dos}^{21,25,26}$. Dambros et al. ${ }^{25}$ sugerem que a companhia de amigos e familiares durante o exercício físico estimula a vida ativa. Além disso, estudos vêm apontando que adolescentes possuem maior envolvimento com a atividade física quando seus pares também estão envolvidos na prática ${ }^{27}$ ou quando há suporte dos amigos ${ }^{28}$.

A segunda barreira mais citada pelos adolescentes foi "não encontro lugares perto de casa com a atividade física que gosto". Apesar de pouco citada na literatura, sabe-se que a qualidade e quantidade dos acessos aos parques e áreas de lazer, estimulam a prática de atividades físicas ${ }^{29}$. Além disso, as áreas públicas de lazer além de escassas ${ }^{30}$ apresentam pouca infraestrutura para a prática de atividade física que atraia os jovens incluindo atividades para além das tradicionalmente consolidadas como os esportes coletivos.

Este estudo apresenta como principal limitação o delineamento transversal dos dados, o que não possibilita o apontamento o sentido de causa e efeito. Com isso os resultados devem ser interpretados com cuidado. Recomenda-se estudos longitudinais que possibilitam maior precisão de associação. Ainda, por conta do número reduzido de adolescentes com idades avançadas, houve um agrupamento (13-16 anos) de forma que a variável apresentasse maior homogeneidade no número de participantes entre as faixas etárias. Essa categorização pode ter incluído adolescentes pré-púberes e púberes, o que pode levar a diferentes percepções de barreiras para a prática de atividade física.

Apesar das limitações supracitadas, os aspectos positivos da seleção de uma escola, possibilitam ações específicas para aquela população. Além disso, o foco da pesquisa em identificar fatores associados a percepção de barreira de insuficientemente ativos, permite planejamento de estratégias específicas para maior envolvimento na prática em atividades físicas de meninos e meninas.

O presente estudo identificou que diferentes barreiras para a prática de atividade física discriminam meninos e meninas insuficientemente ativos. Além disso, fatores que influenciam o envolvimento dos adolescentes em práticas de atividades físicas são distintos entre os sexos. No entanto, a barreira preguiça e o fator baixa percepção de estresse esteve presente em ambos os sexos. Desta forma, ações educacionais devem ser pautadas em estratégias específicas, no sentido de estimular a participação de atividades fiscais entre os adolescentes.

\section{Referências}

1. Huang T, Tarp J, Domazet SL, Thorsen AK, Froberg K, Andersen LB, et al. Associations of Adiposity and Aerobic Fitness with Executive Function and Math Performance in Danish Adolescents. J Pediatr. 2015;167(4):810-5.

2. Huang T, Larsen KT, Jepsen JRM, Møller NC, Thorsen AK, Mortensen EL, et al. Effects of an obesity intervention program on cognitive function in children: A randomized controlled trial. Obesity. 2015;23(10):2101-8.

3. Azevedo MR, Araújo CL, Silva MCd, Hallal PC. Tracking of physical activity from adolescence to adulthood: a population-based study. Rev Saude Publica. 2007;41(1):69-75.

4. Hallal PC, Knuth AG, Cruz DKA, Mendes MI, Malta DC. Prática de atividade física em adolescentes brasileiros. Ciênc Saude Coletiva. 2010;15(2):3035-42.

5. Fermino RC, Rech CR, Hino AAF, Rodriguez Añez CR, Reis RS. Physical activity and associated factors in high-school adolescents in Southern Brazil. Rev Saude Publica. 2010;44(6):986-95.

6. Santos MS, Hino AAF, Reis RS, Rodriguez-Añez CR. Prevalence of barriers for physical activity in adolescents. Rev Bras Epidemiol. 2010;13(1):94-104.

7. Brasil - Instituto Brasileiro de Geografia e Estatística. Pesquisa Nacional de Saúde do Escolar (PeNSE), 2012. 
8. Papalia DE, Feldman RD. Desenvolvimento humano: Artmed Editora; 2013.

9. Azevedo MR, Menezes AM, Assunção MC, Gonçalves H, Arumi I, Horta BL, et al. Tracking of physical activity during adolescence: the 1993 Pelotas Birth Cohort, Brazil. Rev Saude Publica. 2014;48(6):925-30.

10. Cumming SP, Sherar LB, Pindus DM, Coelho-e-Silva MJ, Malina RM, Jardine PR. A biocultural model of maturity-associated variance in adolescent physical activity. Int Rev Sport Exerc Psychol. 2012;5(1):23-43.

11. Finne E, Bucksch J, Lampert T, Kolip P. Age, puberty, body dissatisfaction, and physical activity decline in adolescents. Results of the German Health Interview and Examination Survey (KiGGS). Int J Behav Nutr Phys Act. 2011;8(1):119.

12. Santos MS, Reis RS, Rodriguez-Añez CR, Fermino RC. Desenvolvimento de um instrumento para avaliar barreiras para a prática de atividade física em adolescentes. Rev Bras Ativ Fís Saude. 2009;14(2):76-85.

13. ABEP - Associação Brasileira de Empresas de Pesquisa. Critério de Classificação Econômica Brasil. [citado 2015 nov 24]. Disponível em: http://www.abep.org/new/criterioBrasil.aspx2015

14. Florindo AA, Romero A, Peres SV, Silva MVd, Slater B. Desenvolvimento e validação de um questionário de avaliação da atividade física para adolescentes. Rev Saude Publica. 2006;40(5):802-9.

15. Strong WB, Malina RM, Blimkie CJ, Daniels SR, Dishman RK, Gutin B, et al. Evidence based physical activity for school-age youth. J Pediatr. 2005;146(6):732-7.

16. Pereira ÉF, Moreno C, Louzada FM. Increased commuting to school time reduces sleep duration in adolescents. Chronobiol Int. 2014;31(1):87-94.

17. Drake C, Nickel C, Burduvali E, Roth T, Jefferson C, Badia P. The pediatric daytime sleepiness scale (PDSS): sleep habits and school outcomes in middle-school children. Sleep. 2003;26(4):455-60.

18. Felden ÉP, Carniel JD, Andrade RD, Pelegrini A, Anacleto TS, Louzada FM. Translation and validation of the Pediatric Daytime Sleepiness Scale (PDSS) into Brazilian Portuguese. J Pediatr. 2015;92(2):168-73.

19. Alvarez B, Pavan A. Alturas e comprimentos. Antropometria - técnicas e padronizações. 2007;3:31-44.

20. Goodman E, Adler NE, Kawachi I, Frazier AL, Huang B, Colditz GA. Adolescents' perceptions of social status: development and evaluation of a new indicator. Pediatrics. 2001;108(2):e31-e.

21. Santos MS, Fermino RC, Reis RS, Cassou AC, Añez C. Barreiras para a prática de atividade física em adolescentes. Um estudo por grupos focais. Rev Bras Cineantropom Desempenho Hum. 2010;12(3):137-43.

22. Carskadon MA, Acebo C, Jenni OG. Regulation of adolescent sleep: implications for behavior. Ann N Y Acad Sci. 2004;1021(1):276-91.

23. Santos Legnani RF, Legnani E, da Silva Gasparotto G, Araújo Bacil ED, da Silva MP, de Campos W. Hábitos de sono e prática habitual da atividade física em escolares: uma revisão sistemática. Rev Educ Fís/UEM. 2015;26(1).

24. Gorini MAG, de Souza NA. Avaliação da aprendizagem: a construção de uma proposta em Educação Física. Est Aval Educ. 2007;18(36):181-93.

25. Dambros DD, Lopes LFD, Santos DLd. Barreiras percebidas e hábitos de atividade física de adolescentes escolares de uma cidade do sul do Brasil. Rev Bras Cineantropom Desempenho Hum. 2011;13(6):422-8.

26. Garcia LMT, Fisberg M. Atividades físicas e barreiras referidas por adolescentes atendidos num serviço de saúde. Rev Bras Cineantropom Desempenho Hum. 2011;13(3)163-169.

27. Seabra AF, Mendonça DM, Thomis MA, Anjos LA, Maia JA. Determinantes biológicos e sócio-culturais associados à prática de atividade física de adolescentes Biological and socio-cultural determinants of physical activity in adolescents. Cad Saude Publica. 2008;24:721-36.

28. Van der Horst K, Paw M, Twisk JW, Van Mechelen W. A brief review on correlates of physical activity and sedentariness in youth. Med Sci Sports Exerc. 2007;39(8):1241.

29. Silva DAS, Petroski EL, Reis RS. Barreiras e facilitadores de atividades físicas em freqüentadores de parques públicos. Motriz. 2009;15(2):219-27.

30. Nascimento LCZ, da Silva MR. Juventude e espaços/equipamentos urbanos para o lazer na cidade de Florianópolis. LICERE. 2011;14(4).

ENDEREÇO PARA

CORRESPONDÊNCIA

ÉRICO PEREIRA GOMES FELDEN

email@email.com
CEFID/UDESC

Rua Paschoal Simoni 358

88080-350 Florianópolis - SC, Brasil

Telefone: $48-3321-8600$ 